\title{
Use of the skin prick test and specific immunoglobulin E for the diagnosis of cockroach allergy
}

\author{
Diagnóstico de alergia a baratas no ambiente clínico: \\ estudo comparativo entre o teste cutâneo e IgE específica
}

\author{
Maria Isabella L. Lopes ${ }^{1}$, Paulo J. Miranda², Emanuel Sarinho ${ }^{3}$
}

\section{Resumo}

Objetivos: Comparar a prevalência da sensibilização a baratas (Blattella germanica e Periplaneta americana) em crianças com e sem asma, verificando a concordância entre testes cutâneos e IgE específica bem como determinar a relação entre a sensibilização a baratas e níveis séricos de IgE total.

Métodos: Foi realizado um estudo caso-controle, envolvendo 76 crianças asmáticas e 42 não asmáticas, entre 6 e 14 anos de idade, em Recife, Brasil. Todas as crianças submeteram-se ao teste cutâneo e dosagem sérica de IgE específica para $B$. germanica e $P$. americana e determinação sérica da IgE total.

Resultados: As crianças asmáticas apresentaram maior positividade aos testes cutâneos para B. germanica $(27,6$ versus $4,8 \%)$ e $P$. americana $(27,6$ versus $2,4 \%$ ) que aquelas do grupo controle. A concordância entre o teste cutâneo e a IgE específica foi razoável para B. germanica (Kappa $=0,25)$ e fraca para $P$. americana $($ Kappa $=0,17)$. A média geométrica da IgE total foi $591,70 \mathrm{kU} / \mathrm{L}$ entre pacientes asmáticos e 345,85 kU/L entre os controles, não havendo diferença estatisticamente significante. Em pacientes com testes positivos para baratas, a média geométrica da IgE total foi significativamente maior em comparação aos pacientes cujos exames foram negativos.

Conclusão: A sensibilização a baratas foi associada à asma. 0 teste cutâneo pode refletir mais apropriadamente uma sensibilização a baratas clinicamente relevante que a IgE específica.

J Pediatr (Rio J). 2006;82(3):204-9: Asma, baratas, hipersensibilidade.

\section{Introdução}

As baratas produzem alérgenos potentes que induzem a formação de IgE específica e provocam asma em indivíduos

1. Mestre, Departamento de Pediatria, Universidade Federal de Pernambuco (UFPE), Recife, PE.

2. Mestre, Departamento de Patologia e Imunopatologia, Laboratório Keizo Asami (LIKA), UFPE, Recife, PE.

3. Doutor, Departamento de Pediatria, Divisão de Alergia e Imunologia, UFPE, Recife, PE.

Fonte financiadora: Conselho Nacional de Desenvolvimento Científico e Tecnológico (CNPq).

Artigo submetido em 18.10.05, aceito em 08.03.06.

Como citar este artigo: Lopes MI, Miranda PJ, Sarinho E. Use of the skin prick test and specific immunoglobulin $\mathrm{E}$ for the diagnosis of cockroach allergy. J Pediatr (Rio J). 2006;82:204-9.

\section{Abstract}

Objective: To compare the prevalence of allergy to cockroaches (Blattella germanica and Periplaneta americana) in asthmatic and nonasthmatic children and to determine the degree of agreement between the skin prick test and serum specific IgE levels, as well as to establish the relationship between cockroach allergy and total IgE levels.

Methods: A case-control study involving 76 asthmatic and 42 nonasthmatic children aged between 6 and 14 years was conducted in Recife, Brazil. All individuals were submitted to the skin prick test and to the measurement of specific IgE for $B$. germanica and $P$. americana, as well as to the determination of total IgE concentration.

Results: Asthmatic children showed a higher frequency of positive skin reactions to $B$. germanica ( 27.6 vs. $4.8 \%$ ) and $P$. americana (27.6 vs. $2.4 \%$ ) than non-asthmatic ones. The agreement between the skin prick test and the specific IgE results was reasonable for $B$. germanica $(\mathrm{kappa}=0.25)$ and weak for $P$. americana $(\mathrm{kappa}=0.17)$. Those patients who tested positive for cockroaches had a significantly higher geometric mean for total IgE than those who tested negative. The agreement between specific IgE and the skin prick test increased as total IgE levels rose, although some skin tests were negative even when total IgE levels were as high as $5000 \mathrm{kU} / \mathrm{l}$. All determinations of cockroach-specific IgE were positive for total IgE levels greater than $2500 \mathrm{kU} / \mathrm{l}$, even among asymptomatic patients.

Conclusion: Allergic sensitivity to cockroaches was a predictive factor for asthma severity. The skin prick test is more appropriate for the detection of clinically relevant sensitivity to cockroaches than specific IgE determination.

J Pediatr (Rio J). 2006;82(3):204-9: Asthma, cockroaches, hypersensitivity.

geneticamente suscetíveis, quando expostos ${ }^{1}$. As espécies domésticas mais comuns de baratas são a Blattella germanica e a Periplaneta americana, encontradas no mundo todo, em condições favoráveis para sua proliferação, tais como clima quente e úmido e condições precárias de moradia $^{1}$.

A sensibilização a baratas entre indivíduos asmáticos ou com rinite varia consideravelmente. Freqüências de sensibilização de até $70 \%$ foram relatadas em algumas cidades da América do Norte ${ }^{2}$. Entretanto, a alergia a baratas é menos comum na Europa ${ }^{2-4}$. No Brasil, existem poucos estudos sobre a exposição e sensibilização a baratas. Em algumas cidades, a positividade de testes cutâneos para $B$. germanica e/ou P. americana chega a $55 \%{ }^{5}$. 
Os alérgenos produzidos pelas baratas são encontrados na poeira doméstica em colchões, roupas de cama, móveis estofados, carpetes e principalmente na cozinha6-8. Devido à natureza perene da exposição, - o que não permite estabelecer uma relação temporal em relação ao desenvolvimento de sintomas - o diagnóstico da alergia a baratas com base apenas em dados clínicos é inviável, necessitando-se, assim, de testes complementares ${ }^{9}$.

A sensibilização a alérgenos é diagnosticada através de testes in vivo, tais como testes cutâneos para determinação de hipersensibilidade imediata, e através de técnicas in vitro, como a dosagem da IgE específica ${ }^{10}$. Apesar dos avanços nessa área, não existe nenhum padrão-ouro para a detecção de IgE específica para todos os alérgenos inalantes ${ }^{9-11}$. Isso é ainda mais complicado quando se trata do diagnóstico de sensibilização a baratas, pois atualmente não existem extratos comerciais padronizados para esses alérgenos. O uso de extratos padronizados com potência conhecida estabelecida resultaria em respostas mais reprodutíveis, com um menor número de falsos positivos ${ }^{11}$. No futuro, alérgenos recombinantes serão usados para um diagnóstico mais preciso de alergias específicas ${ }^{11,12}$.

Os principais objetivos deste estudo são comparar a prevalência de sensibilização a baratas em crianças com e sem asma e avaliar a concordância entre o teste cutâneo e a IgE específica no diagnóstico de sensibilização a baratas. Além disso, será avaliada a relação entre sensibilização a baratas e os níveis da IgE total.

\section{Métodos}

O estudo foi realizado em Recife, Brasil. Recife é uma cidade com clima tropical, o que predispõe à proliferação de baratas, especialmente em moradias de condições precárias. O grupo de estudo incluiu crianças com idade entre 6 e 14 anos, selecionadas no ambulatório de alergia e imunologia pediátrica do Serviço de Pediatria Geral do Hospital das Clínicas da Universidade Federal de Pernambuco, entre abril e agosto de 2001 . O estudo foi aprovado pelo Comitê de Ética em Pesquisa do Centro de Ciências da Saúde, Universidade Federal de Pernambuco, e obteve-se um consentimento informado de todos os participantes do estudo.

\section{Delineamento do estudo}

Foi realizado um estudo caso-controle a fim de comparar a prevalência da sensibilização a baratas em crianças com e sem asma. Os indivíduos eram considerados asmáticos (casos) se apresentassem no mínimo três episódios de dispnéia nos últimos 12 meses ou se tivessem diagnóstico clínico de asma e estivessem em uso de profilaxia. As crianças que não possuíam histórico de dispnéia, sibilância, rinite alérgica ou dermatite atópica foram incluídas no grupo de não-asmáticos (controle). Os pacientes foram selecionados a partir do ambulatório de pediatria através de amostras aleatórias, e foram distribuídos nos grupos de acordo com os critérios de inclusão. O tamanho da amostra foi calculado pelo software Epi-Info 6.0, e a proporção entre casos e controles foi de $2: 1$, totalizando 76 casos e 42 controles. Todas as crianças foram submetidas ao teste cutâneo e à dosagem da IgE total e específica para $B$. germanica e $P$. americana.

\section{Testes cutâneos}

Os testes cutâneos foram realizados com extratos de $B$. germanica ( $1: 10$ peso/volume) e $P$. americana ( $1: 10$ peso/ volume), através da técnica de punção. Uma solução histamínica básica $(10 \mathrm{mg} / \mathrm{ml})$ foi usada como controle positivo e uma solução de glicerina, como controle negativo. Os extratos e os controles foram obtidos do laboratório Hollister-Stier, Spokane, Washington. Para garantir a uniformidade, os mesmos lotes de extratos de barata foram usados em todos os indivíduos, apenas um pesquisador realizou os testes cutâneos, e o mesmo tipo de dispositivo foi usado no teste de punção. O teste foi considerado positivo quando a pápula (wheal) era no mínimo $3 \mathrm{~mm}$ maior que no controle negativo ${ }^{11,12}$.

\section{IgE sérica específica e total}

Coletou-se sangue venoso de cada paciente. As amostras foram centrifugadas a $3500 \mathrm{rpm}$ e $1 \mathrm{ml}$ do soro foi armazenado a $-20^{\circ} \mathrm{C}$ para posterior determinação da IgE total e IgE específica para B. germanica e $P$. americana. A IgE total e a IgE específica foram medidas com UniCAP ${ }^{\circledR} /$ Pharmacia CAP System ${ }^{\mathrm{TM}}$, de acordo com as instruções do fabricante. Um indivíduo era considerado sensibilizado quando os níveis de IgE específica eram maiores que $0,35 \mathrm{kU}_{\mathrm{A}} / \mathrm{I}$ (classe 1).

\section{Análise estatística}

Os dados obtidos foram analisados através do software Epi-Info (versão 6.0). Foi feita uma análise bivariada para identificar a possível associação entre a sensibilização ao alérgeno de barata e a asma. A força dessa associação foi avaliada pelo cálculo da razão de probabilidade (ou odds ratio $=\mathrm{OR}$ ). $\mathrm{O}$ teste do qui-quadrado foi empregado juntamente com a correção de Yates ou teste exato de Fischer, sempre que necessário, a fim de avaliar a significância estatística, onde um valor de $p<0,05$ foi considerado significativo. O coeficiente Kappa foi usado para avaliar a concordância entre os resultados dos testes cutâneos e a IgE específica. Os níveis séricos de IgE foram comparados através da média geométrica. Para avaliar a variação na proporção de pacientes com IgE específica e teste cutâneo positivos em relação à IgE total, aplicou-se um modelo de regressão logística simples, no qual a variável explicativa foi o logaritmo de Napier para a IgE total.

\section{Resultados}

A maioria dos indivíduos de ambos os grupos é proveniente da região metropolitana de Recife, com uma renda per capita mensal de R\$120,00 (US\$ 60) ou menos. Outras características que podem influenciar o nível de exposição a baratas são apresentadas na Tabela 1. 
Tabela 1 - Variáveis sociais e demográficas que podem influenciar a exposição a baratas em asmáticos e controles

\begin{tabular}{|c|c|c|c|c|c|c|c|}
\hline \multirow[b]{2}{*}{ Variáveis demográficas } & \multicolumn{2}{|c|}{ Asmáticos } & \multicolumn{2}{|c|}{ Controles } & \multicolumn{2}{|c|}{ Total } & \multirow[t]{2}{*}{$\mathbf{p}$} \\
\hline & $\mathbf{n}$ & $\%$ & $\mathbf{n}$ & $\%$ & $\mathbf{n}$ & $\%$ & \\
\hline \multicolumn{8}{|l|}{ Idade (anos) } \\
\hline $6-9$ & 43 & 56,6 & 26 & 61,9 & 69 & 58,5 & \multirow{2}{*}{$\begin{array}{c}\chi^{2} \text { Yates }=0,1 \\
p=0,713\end{array}$} \\
\hline $10-14$ & 33 & 43,4 & 16 & 38,1 & 49 & 41,5 & \\
\hline \multicolumn{8}{|l|}{ Gênero } \\
\hline Feminino & 27 & 35,5 & 14 & 33,3 & 41 & 34,7 & \multirow{2}{*}{$\begin{array}{c}\chi^{2} \text { Yates }=0,00 \\
p=0,97\end{array}$} \\
\hline Masculino & 49 & 64,5 & 28 & 66,7 & 77 & 65,3 & \\
\hline \multicolumn{8}{|l|}{ Fornecimento de água } \\
\hline Moradias com água encanada & 60 & 78,9 & 38 & 90,5 & 98 & 83,1 & \multirow{2}{*}{$\begin{array}{c}\chi^{2} \text { Yates }=1,80 \\
p=0,18\end{array}$} \\
\hline Outros & 16 & 21,1 & 4 & 9,5 & 20 & 16,9 & \\
\hline \multicolumn{8}{|l|}{ Sistema de esgoto } \\
\hline Público & 62 & 81,6 & 30 & 71,4 & 92 & 78 & \multirow{3}{*}{ Fisher $\mathrm{p}=0,49$} \\
\hline Sem esgoto & 9 & 11,8 & 2 & 4,8 & 11 & 9 & \\
\hline Informações não disponíveis & 5 & 6,6 & 10 & 23,8 & 15 & 13 & \\
\hline \multicolumn{8}{|l|}{ Tratamento do lixo } \\
\hline Coleta por companhia pública & 68 & 89,5 & 40 & 95,2 & 108 & 91,5 & \multirow{2}{*}{$\begin{array}{c}\chi^{2} \text { Yates }=0,53 \\
p=0,45\end{array}$} \\
\hline Queimado/enterrado/abandonado & 8 & 10,5 & 2 & 4,8 & 10 & 8,5 & \\
\hline
\end{tabular}

\section{Testes cutâneos}

Houve positividade no teste cutâneo para $B$. germanica em $21(27,6 \%)$ crianças asmáticas, comparativamente a apenas duas $(4,8 \%)$ crianças no grupo controle $(\mathrm{OR}=7,64$; IC95\% 1,57-50,75; $p=0,006)$. Foi observada positividade para $P$. americana em $21(27,6 \%)$ crianças asmáticas e em uma $(2,4 \%)$ criança do grupo controle (OR = 15,65; IC95\% $2,05-330,51 ; p=0,002)$. No teste cutâneo, vinte e seis $(34,2 \%)$ crianças asmáticas e duas $(4,8 \%)$ crianças do grupo controle mostraram sensibilização a pelos menos uma das espécies de baratas (OR $=10,40$; IC95\% 2,16$68,45 ; p<0,001)$.

\section{IgE específica para baratas}

Encontrou-se IgE sérica específica para $B$. germanica acima de $0,35 \mathrm{kU}_{\mathrm{A}} / \mathrm{l}$ em $32(42,1 \%)$ crianças asmáticas e em nove $(21.4 \%)$ crianças do grupo controle $(\mathrm{OR}=2,67$; IC95\% 1,03-7,03; $\mathrm{p}=0,04)$. Quanto à $P$. americana, a IgE específica foi positiva em $22(28,9 \%)$ crianças asmáticas e em sete $(16,7 \%)$ crianças do grupo controle $(O R=2,04$; IC95\% 0,72-5,96; $p=0,21)$. Trinta e três (43,3\%) crianças asmáticas e $10(23,8 \%)$ crianças do grupo controle apresentaram IgE específica positiva a pelo menos uma das espécies de barata $(O R=2,46$; IC95\% 0,98-6,29; $p=0,06)$.

\section{Concordância entre o teste cutâneo e a IgE específica}

Ambos os testes demonstraram concordância (positiva ou negativa) quanto ao diagnóstico de sensibilização à $B$. germanica em 70\% (82 de 118) dos casos. Destes, $12 \%$ (14 de 118 ) foram positivos e $58 \%$ (68 de 118 ), negativos. Quanto à $P$. americana, a concordância geral foi de $72 \%$ ( 85 de 118 ), sendo $8 \%$ (9 de 118 ) positivos e $64 \%$ (76 de 118 ), negativos.

A concordância observada entre o teste cutâneo e a IgE específica, determinada pelo coeficiente Kappa, foi razoável para B. germanica (Kappa $=0,25$ ) e fraca para $P$. americana (Kappa $=0,17)$.

\section{IgE total}

A média geométrica para a IgE total foi de $591,70 \mathrm{kU} / \mathrm{I}$ nos pacientes asmáticos e de $345,85 \mathrm{kU} / \mathrm{I}$ nas crianças do grupo controle, sem significância estatística entre os dois grupos (teste $t$ de Student sem correção de Welch, $p=0,08)$.

\section{Relação entre o teste cutâneo e a IgE total}

A média geométrica para a IgE total foi significativamente maior $(989,4 \mathrm{kU} / \mathrm{l})$ entre os pacientes com testes cutâneos positivos para baratas (B. germanica e/ou $P$. americana) do que entre aqueles cujos testes cutâneos foram negativos $(392,4 \mathrm{kU} / \mathrm{I})(\mathrm{p}=0,002$; teste $t$ de Student para amostras independentes).

A proporção de pacientes com testes cutâneos positivos para $B$. germanica e/ou $P$. americana cresceu com o aumento na IgE total. Isso foi evidenciado através do ajuste de um modelo de regressão logística simples no qual a variável explicativa foi o logaritmo de Napier para a IgE total. A 
Figura 1 mostra a variação entre as proporções estimadas dos testes cutâneos, de acordo com o logaritmo de Napier, e a IgE total.

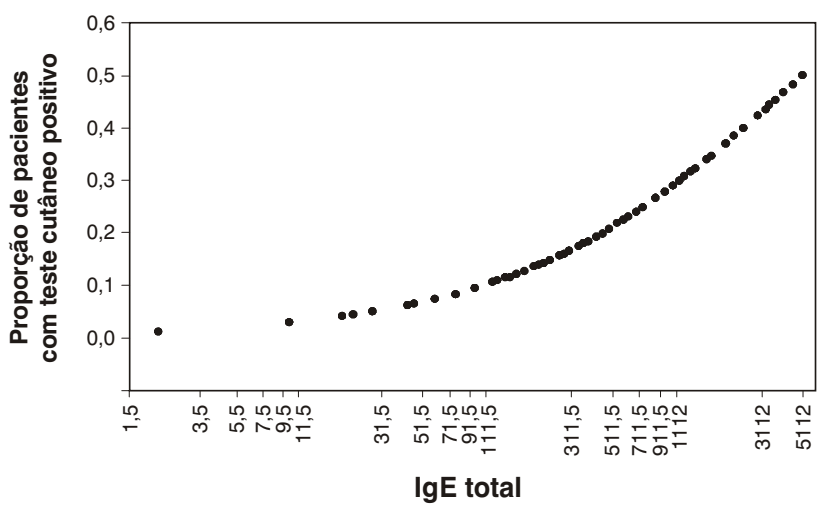

Figura 1 - Variação entre as proporções estimadas de testes cutâneos positivos, de acordo com o logaritmo de Napier, e a IgE total

\section{Relação entre IgE total e IgE específica}

A média geométrica para a IgE total também foi significativamente maior $(1255,0 \mathrm{kU} / \mathrm{l})$ no grupo de pacientes com IgE específica positiva para baratas (B. germanica e/ou $P$. americana) em comparação ao grupo que apresentou IgE específica negativa $(284,6 \mathrm{kU} / \mathrm{l})(\mathrm{p}<0,001$; teste $t$ de Student para amostras independentes).

O número de crianças com IgE específica positiva para B. germanica e/ou $P$. americana cresceu proporcionalmente ao aumento da IgE total, de forma semelhante ao que ocorreu com os testes cutâneos. Novamente, usou-se um modelo de regressão logística simples ajustado, no qual a variável explicativa foi o logaritmo de Napier para a IgE total. A Figura 2 mostra a variação entre as proporções estimadas da IgE específica, de acordo com o logaritmo de Napier, e a IgE total.

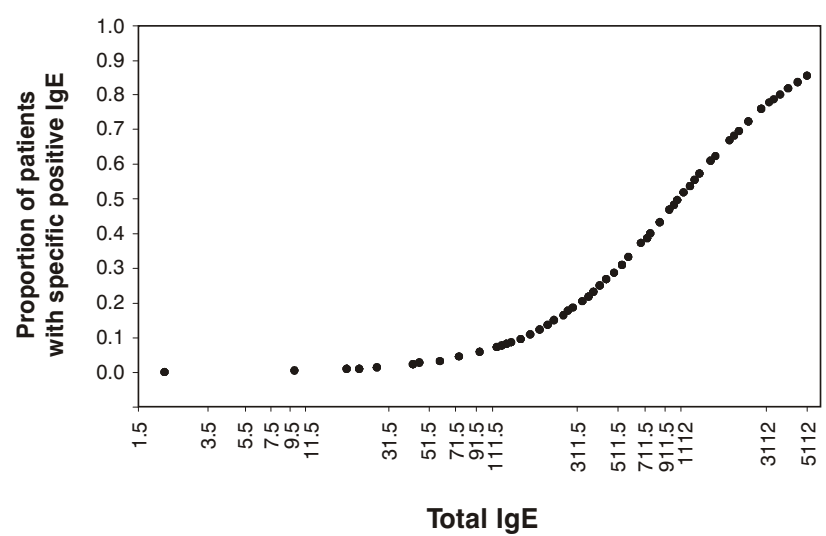

Figura 2 - Variação entre as proporções estimadas de IgE específica positiva, de acordo com o logaritmo de Napier, e a IgE total

\section{Discussão}

Não existe um padrão-ouro para avaliar a acurácia do diagnóstico clínico de alergia a baratas. Em nosso estudo, os testes cutâneos para $B$. germanica e $P$. americana foram positivos em $27,6 \%$ das crianças asmáticas, com resultados semelhantes ao de outro estudo brasileiro ${ }^{13}$. Houve uma associação significativa entre a sensibilização a ambas espécies de baratas e a asma; entretanto, devido ao pequeno tamanho da amostra, a razão de probabilidade (OR) apresentou amplos intervalos de confiança, que impediram uma estimativa precisa da força dessa associação.

Quando a sensibilização a baratas foi determinada pela IgE específica, observamos que, comparativamente aos testes cutâneos, a freqüência de sensibilização a $B$. germanica foi maior tanto nos asmáticos $(42,1 \%)$ quanto nos controles $(21,4 \%)$. A percentagem de sensibilização à $P$. americana no grupo de crianças asmáticas, determinada tanto via teste cutâneo quanto via IgE específica ( 27,6 versus $28,9 \%$, respectivamente), foi equivalente, porém mais pacientes no grupo controle apresentaram resultados positivos para a $\operatorname{IgE}(16,7 \%)$ do que nos testes cutâneos $(2,4 \%)$. Considerando o grande número de pacientes do grupo controle que apresentaram resultados positivos para a $P$. americana, a associação entre a sensibilização a baratas e a asma foi significativa apenas para a $B$. germanica. A falta de associação entre a IgE específica positiva para $P$. americana e asma neste estudo pode ser oriunda das diferenças de sensibilização e especificidade do imunoensaio para esse antígeno, e/ou do fato de que o tamanho da amostra não foi suficiente para detectar uma diferença significativa entre os casos e controles.

A alta percentagem de IgE específica positiva observada nos controles deste estudo está de acordo com aquela encontrada em publicações recentes. Em um estudo taiwanês, por exemplo, $43 \%$ das crianças asmáticas e $20 \%$ das não-asmáticas eram sensíveis à $B$. germanica ${ }^{14}$. Em outro estudo caso-controle realizado em Gana, o teste cutâneo foi positivo em $30 \%$ dos asmáticos e $10 \%$ dos controles, enquanto que a IgE específica foi positiva em $76 \%$ dos asmáticos e $28 \%$ dos controles ${ }^{15}$. Tradicionalmente, os testes para determinar a IgE específica são considerados menos sensíveis que os testes cutâneos, mas recentes avanços nas técnicas de imunoensaio ${ }^{16}$ talvez melhorem o desempenho desses ensaios, que podem justificar essa maior sensibilidade. Todavia, o problema de perda de especificidade permanece.

Neste estudo, a concordância observada entre o teste cutâneo e a IgE específica através do coeficiente Kappa foi fraca para $P$. americana $(0,17)$ e razoável para $B$. germanica $(0,25)$. Esses resultados não se deveram ao ponto de corte utilizado para definir a sensibilização, já que quando este foi alterado para classe $2\left(\geq 0,7 \mathrm{kU}_{\mathrm{A}} / \mathrm{I}\right)$, a concordância foi ainda pior: fraca para $B$. germanica $(0,15)$ e $P$. americana $(0,10)$. Maia et al. também não observaram concordância entre os dois testes na sensibilização à $B$. germanica ${ }^{17}$. Sastre et al. encontraram uma concordância de apenas $21 \%$ entre o teste cutâneo e a IgE específica para B. germanica ${ }^{5}$. 
A discordância entre os testes cutâneos e a IgE específica também pode resultar do uso de extratos não padronizados, que apresentam potência variável e resultados menos reprodutíveis. Esses extratos podem estar contaminados com outras proteínas, alérgenos e enzimas ${ }^{11}$. Estudos que utilizam extratos padronizados de alérgenos inalantes demonstram maior concordância entre os testes cutâneos e a IgE específica ${ }^{18,19}$.

Os alérgenos recombinantes contêm concentrações bem definidas dos principais alérgenos, apresentando maior especificidade e menos problemas com reações cruzadas falsas $^{20}$. Assim que tais reagentes forem disponibilizados para a avaliação da sensibilização a baratas, provavelmente haverá maior concordância entre os testes cutâneos e a IgE específica. Outros fatores que influenciam os resultados dos testes cutâneos são a potência do extrato, o tipo de dispositivo usado para a punção, a habilidade de quem realiza os testes, a acurácia na interpretação dos resultados e a possibilidade de reações cruzadas. Existem limitações quanto ao uso de medicação, problemas dermatológicos e idade do paciente, mas mesmo assim, ainda é o método de escolha para a análise de alérgenos inalantes ${ }^{21}$.

O erro na dosagem sérica da IgE específica pode ocorrer devido ao tipo de alérgeno usado, à quantidade insuficiente de alérgenos disponíveis, à destruição do epítopo durante a fixação na fase sólida, à ligação insuficiente à $\mathrm{IgE}$, aos níveis de IgG (que podem competir com a IgE, produzindo falsos negativos), aos níveis elevados de IgE total (que também podem produzir falsos positivos devido à ligação nãoespecífica $)^{12,16}$, à presença de IgE específica para determinantes de carboidratos ${ }^{22}$ e a outras reações cruzadas ${ }^{23}$. Ao se analisar todas as amostras para a dosagem sérica de $\mathrm{IgE}$ específica, recomenda-se determinar também os níveis de IgE total, pois quanto maiores estes últimos, maior será o grau de ligação não-específica. Sugere-se investigar os resultados positivos de IgE sérica específica em pacientes com IgE total extremamente alta através de ensaios de inibição alergênica ${ }^{12}$.

Em um estudo no qual compararam o desempenho do teste cutâneo e a IgE específica com um modelo de provocação alergênica nasal com o uso de alérgenos recombinantes de pólen, Niederberger et al. observaram que o teste cutâneo foi mais eficiente na previsão do potencial alergênico em relação à indução de sintomas respiratórios do que a sorologia quantitativa, já que não havia uma boa correlação entre os níveis de IgE específica e os testes biológicos ${ }^{24}$.

O nível de IgE sérica total é freqüentemente usado para o diagnóstico de atopia, mas seu uso clínico é limitado devido à grande sobreposição de valores de IgE em indivíduos atópicos e não-atópicos, além da existência de uma série de distúrbios que elevam os níveis de IgE total, incluindo infecção helmíntica intestinal ${ }^{25}$. No presente estudo, não houve diferença estatisticamente significativa entre a média geométrica da IgE total nos asmáticos $(591,7 \mathrm{kU} / \mathrm{l})$ e nos controles $(345,85 \mathrm{kU} / \mathrm{l})$. O que chamou a nossa atenção foram os níveis elevados de IgE total em ambos os grupos. Aproximadamente $34 \%$ dos asmáticos e controles apresentaram níveis de IgE total iguais a ou acima de $1000 \mathrm{kU} / \mathrm{l}$. Níveis de IgE total iguais a ou acima de $3000 \mathrm{kU} / \mathrm{l}$ foram encontrados com maior freqüência entre os controles (12\%) do que entre os asmáticos (9\%).

Os altos níveis de IgE total podem ser causados por infecções helmínticas, o que torna a relação entre asma, atopia e parasitismo intestinal complexa ${ }^{26}$. Em uma pesquisa realizada com 150 pré-escolares da cidade de Natal, Brasil, Sales et al. encontraram uma alta percentagem $(88,6 \%)$ de ascaridíase, com níveis de IgE total significativamente maiores entre as crianças infectadas (média geométrica $=715 \mathrm{UI} / \mathrm{ml}$ ), em comparação às crianças não infectadas (média geométrica $=72,8 \mathrm{UI} / \mathrm{ml}$ ) ${ }^{27}$. Nessas crianças, a ascaridíase e os testes cutâneos positivos foram fortemente associados à sibilância.

Os elevados níveis de IgE total nos asmáticos e controles do nosso estudo podem ter sido causados por parasitoses intestinais, devido às baixas condições socioeconômicas de nossos pacientes, o que os torna suscetíveis a esse tipo de infecção. Outras explicações para o diagnóstico de sensibilização assintomática no grupo controle incluem também a inibição de manifestações alérgicas devido à saturação dos mastócitos ${ }^{28}$, falsos positivos devido à ligação não-específica em razão dos altos níveis de $\operatorname{IgE}^{12}$ ou reações cruzadas com alérgenos de parasitas intestinais, especialmente Ascaris, que é bastante prevalente na área estudada.

A média geométrica para a IgE total em pacientes com testes cutâneos positivos para $B$. germanica e/ou $P$. americana foi aproximadamente 2,5 vezes maior que aquela dos indivíduos que apresentaram resultados negativos, com especial atenção para o fato de que a proporção de testes positivos aumentava à medida os níveis de IgE total cresciam. Huss et al. observaram que a IgE total em crianças asmáticas representava um fator de risco para testes cutâneos com resultados positivos para baratas e ácaros da poeira domiciliar, principalmente quando os níveis encontravam-se acima de $869 \mathrm{ng} / \mathrm{ml}$ (362 UI/ml) 29 .

Uma tendência semelhante foi observada ao se analisar a relação entre os níveis de IgE específica e IgE total. A média geométrica para a IgE total foi aproximadamente 4,5 vezes maior entre os pacientes sensíveis do que entre aqueles que não mostraram sensibilização. Observamos que a proporção de IgE específica positiva também aumentou à medida que a IgE total elevou-se, e acima de $2.500 \mathrm{kU} / \mathrm{I}$ de IgE total, todas as dosagens de IgE específica para baratas foram positivas, mesmo nos pacientes assintomáticos. De acordo com nossos dados, um estudo com mulheres da Tanzânia revelou uma correlação positiva entre a IgE total e a IgE específica para ácaros da poeira e baratas, embora ainda não associada à asma, nem aos níveis de exposição a alérgenos ${ }^{30}$. Uma das explicações para esses achados foi a saturação dos receptores de IgE causada pelo elevado nível de IgE total que bloqueia o acesso da IgE específica aos mastócitos, suprimindo assim a resposta alérgica. Os altos níveis de IgE total se deveram provavelmente a uma infecção helmíntica intestinal. Uma reação cruzada entre parasitos, ácaros da poeira e baratas pode ser outra explicação para os resultados obtidos ${ }^{30}$. É possível que, em nosso estudo, os resultados positivos para 
a IgE específica encontrados em pacientes com altos níveis de IgE total estejam relacionados à ativação policlonal dos linfócitos B devido à infecção helmíntica intestinal ou à reação cruzada entre os vários alérgenos.

O estudo comparativo entre o teste cutâneo e a IgE específica para o diagnóstico de alergia a baratas sugere que o teste cutâneo pode refletir melhor uma sensibilização clinicamente relevante a baratas. Contudo, são necessários estudos com tamanho amostral maior para investigar essa questão.

\section{Agradecimentos}

Nossos agradecimentos ao Dr. João Bosco de Oliveira Filho por sua análise crítica do artigo.

\section{Referências}

1. Chapman MD, Vailes LD, Hayden ML, Benjamin DC, Platts-Mills TA, Arruda LK. Structural and antigenic studies of cockroach allergens and their relevance to asthma. Adv Exp Med Biol. 1996;409:95-101.

2. Hirsch T, Stappenbeck C, Neumeister V, Weiland SK, Von Mutius E, Keil U, et al. Exposure and allergic sensitization to cockroach allergen in East Germany. Clin Exp Allergy. 2000;30:529-37.

3. Peruzzi M, Luca M, Novembre $E$, Martino M, Vierucci A. Incidence of cockroach allergy in atopic Italian children. Ann Allergy Asthma Immunol. 1999;83:167-71.

4. Sastre J, Ibañez MD, Lombardero M, Laso MT, Lehrer S. Allergy to cockroaches in patients with asthma and rhinitis in an urban area (Madri). Allergy. 1996;51:582-6.

5. Santos ABS, Chapman MD, Aalberse RC, Vailes LD, Ferriani VPL, Oliver $C$, et al. Cockroach allergens and asthma in Brazil: identification of tropomyosin as a major allergen with potential cross-reactivity with mite and shrimp allergens. J Allergy Clin Immunol. 1999;104:329-37.

6. De Lucca SD, Taylor DJ, O'Meara TJ, Jones AS, Tovey ER. Measurement and characterization of cockroach allergens detected during normal domestic activity. J Allergy Clin Immunol. $1999 ; 104: 672-80$.

7. Eggleston PA, Rosenstreich D, Lynn H, Gergen P, Baker D, Kattan $M$, et al. Relationship of indoor allergen exposure to skin test sensitivity in inner-city children with asthma. J Allergy Clin Immunol. 1998;102:563-70.

8. de Blay F, Sanchez J, Hedelin G, Perez-Infante A, Verot A, Chapman $M$, et al. Dust and airborne exposure to allergens derived from cockroach (Blattella germanica) in low-cost public housing in Strasbourg (France). J Allergy Clin Immunol. 1997; 99: 107-12.

9. Current issues relating to in vitro testing for allergen-specific IgE: a workshop report. Ann Allergy Asthma Immunol. 1999;82: 407-12.

10. Homburguer HA. Methods in laboratory immunology. In: Middleton E, Reed CE, editors. Allergy: principles \& practice. 5th ed. St. Louis: Mosby-Year Book; 1998. p. 417-29.

11. The use of standardized allergen extracts. American Academy of Allergy, Asthma and Immunology (AAAAI). J Allergy Clin Immunol. 1997;99:583-6.

12. Bernstein IL, Storms WW. Practice parameters for allergy diagnostic testing. Joint Task Force on Practice Parameters for the Diagnosis and Treatment of Asthma. The American Academy of Allergy, Asthma and Immunology and the American College of Allergy, Asthma and Immunology. Ann Allergy Asthma Immunol. 1995;75:543-625.
13. Rosário Filho NA, Faria L, Riedi CA, Zulato S. Sensibilização a baratas em crianças asmáticas: relação com a gravidade da doença. Rev Bras Alerg Imunopatol. 1999;22:151-5.

14. Lin YC, Su HJ, Hsiue TR, Lee CH, Chen CW, Guo YL. Levels of house dust mite-specific IgE and cockroach-specific IgE and their association with lower pulmonary function in Taiwanese children. Chest. 2002;121:347-53.

15. Addo-Yobo EO, Custovic A, Taggart SC, Craven M, Bonnie B, Woodcock A. Risk factors for asthma in urban Ghana. J Allergy Clin Immunol. 2001;108:363-8.

16. Yunginger JW, Ahlstedt $S$, Eggleston PA, Homburguer $H A$, Nelson HS, Ownby DR, et al. Quantitative IgE antibody assays in allergic diseases. J Allergy Clin Immunol. 2000;105:1077-84.

17. Maia AAM, Croce J, Guimarães JH, Lopez M. Sensibilização alérgica à Blattella germanica (Insecta: Dyctyoptera) em pacientes com asma e rinite na cidade de São Paulo, Brasil. Rev Bras Alerg Imunopatol. 1996;19:47-50.

18. Sarinho E, Rizzo MC, Just E, Fernandez-Caldas E, Solé D. Sensibilização aos ácaros domésticos em crianças atópicas e não atópicas do Recife, PE, Brasil. Rev Bras Alerg Imunopatol. 2000;23:105-10.

19. Wood RA, Phipatanakul W, Hamilton RG, Eggleston PA. A comparison of skin prick tests, intradermal tests, and RASTs in the diagnosis of cat allergy. J Allergy Clin Immunol. 1999;103: 773-9.

20. Chapman MD, Smith AM, Vailes MD, Arruda LK, Dhanaraj V, Pomés A. Recombinant allergens for diagnosis and therapy of allergic disease. J Allergy Clin Immunol. 2000;106:409-18.

21. Wood RA. The diagnosis of allergy: why is it so difficult? Ann Allergy Asthma Immunol. 2003;91:1-2.

22. Mari A, Iacovacci $P$, Afferni $C$, Barletta $B$, Tinghino R, Di Felice $\mathrm{G}$, et al. Specific IgE to cross-reactive carbohydrate determinants strongly affect the in vitro diagnosis of allergic diseases. J Allergy Clin Immunol. 1999;103:1005-11.

23. Aalberse RC. Allergens from mites: implications of cross-reactivity between invertebrate antigens. Allergy. 1998;53:47-8.

24. Niederberger V, Stübner $P$, Spitzauer S, Kraft D, Valenta R, Ehrenberger $K$, et al. Skin test results but not serology reflect immediate type respiratory sensitivity: a study performed with recombinant allergen molecules. J Invest Dermatol. 2001;117: 848-51.

25. Ownby DR. Clinical significance of immunoglobulin E. In: Middleton E, Reed CE, editors. Allergy: principles \& practice. 5th ed. St. Louis: Mosby-Year Book; 1998. p. 770-82.

26. Lynch NR, Goldblatt J, Le Souëf PN. Parasite infections and the risk of asthma and atopy. Thorax. 1999;54:659-60.

27. Sales VS, Rodrigues CE, Cavalcanti GB, Trombone APF, Lima $R C$, Santos $A B R$, et al. Infection with Ascaris lumbricoides in preschool children: role in wheezing and IgE responses to inhalant allergens. J Allergy Clin Immunol. 2002;109:S27.

28. Weiss ST. Parasites and asthma/allergy: what is the relationship? J Allergy Clin Immunol. 2000;105:205-10.

29. Huss K, Adkinson NF Jr, Eggleston PA, Dawson C, Van Natta ML, Hamilton RG. House dust mite and cockroach exposure are strong risk factors for positive allergy skin test responses in the Childhood Asthma Management Program. J Allergy Clin Immunol. 2001;107:48-54.

30. Sunyer J, Torregrosa J, Anto JM, Menedez C, Acosta C, Schellenberg $D$, et al. The association between atopy and asthma in a semirural area of Tanzania (East Africa). Allergy. 2000;55:762-6.

\section{Correspondência:}

Emanuel Sarinho

Av. Parnamirim, 327/202, Parnamirim

CEP 52060-000 - Recife, PE

Tel.: (81) 8881.4801

Fax: (81) 3231.2122

E-mail: emanuel.sarinho@gmail.com 